\title{
Construcción de la maternidad en adopciones monoparentales: mandatos, deseos y elecciones
}

\author{
Motherhood Construction in Single Parent Adoptions: \\ Mandates, Desires and Choices
}

\author{
Irene Salvo Agoglia \\ Universidad Central de Chile, Santiago, Chile
}

\begin{abstract}
Resumen: En este artículo se presentan algunos resultados de una investigación cualitativa más amplia que describió y analizó las narrativas de mujeres que adoptaron sin pareja en Chile durante los años 1999-2012. El trabajo pretende hacer foco en el análisis de las continuidades, rupturas e innovaciones en las significaciones y prácticas de estas mujeres, respecto de algunos mandatos asociados al género femenino. Los principales resultados se organizan en tres núcleos narrativos ligados a la construcción identitaria, la construcción de la maternidad y la decisión de adoptar, mostrando la coexistencia de diversos sentidos y prácticas y las estrategias y acciones que realizan para gestionar sus decisiones y vidas familiares. Se discuten y problematizan las nociones de deseo, autonomía, elección y agencia con el objetivo de contribuir a profundizar en el conocimiento y comprensión de algunas nociones y procesos asociados a estas y otras maternidades, para orientar actuaciones profesionales reflexivas, pertinentes y desprejuiciadas que lleven a repensar nociones más amplias sobre la maternidad y el parentesco, intentando aportar con ello a legitimar modalidades familiares contemporáneas y emergentes.
\end{abstract}

Palabras clave: adopciones monoparentales, narrativas, mandatos, deseos, elecciones.

\begin{abstract}
This article presents some of the results of a qualitative research aimed to describe and analyze the narratives of women who adopted without a partner in Chile between the years 19992012. The paper aims to focus on the analysis of the continuities, ruptures and innovations in the meanings and practices of these women, regarding some mandates associated with the feminine gender. The main results are organized in three narrative axes linked to the construction of identity, the construction of motherhood and decision to adopt, showing the coexistence of different senses and practices, and the strategies and actions that they perform to manage their decisions and family lives. The notions of desire, autonomy, choice and agency are discussed and problematized with the aim of contributing to deepen the knowledge and understanding of some notions and processes associated with these and other motherhoods, and to guide reflective and unprejudiced professional actions that lead to rethink broader notions about motherhood and kinship, contributing to legitimize contemporary and emerging family patterns.
\end{abstract}

Keywords: single parent adoptions, narratives, mandates, desires, choices.

La autora agradece el financiamiento de la presente investigación a la Beca Chile de Doctorado en el Extranjero (2010-2014), otorgada por la Comisión Nacional de Investigación Científica y Tecnológica (Conicyt) de Chile y al Fondo Interno de Investigación de la Universidad Alberto Hurtado, con el cual fue posible financiar los gastos operacionales asociados al trabajo de campo, así como también a las instituciones que colaboraron en el estudio y, muy especialmente, a las participantes que aceptaron ser entrevistadas y relatar sus experiencias de vida. Finalmente, la autora agradece a los/las dos evaluadores/as externos/as anónimos/as que con sus comentarios contribuyeron a mejorar el presente texto.

Contacto: I. Salvo Agoglia. Lord Cochrane 417, Santiago, Chile. Correo electrónico: irene.salvo@ucentral.cl

Cómo citar: Salvo Agoglia, I. (2016). Construcción de la maternidad en adopciones monoparentales: mandatos, deseos y elecciones. Revista de Psicología, 25(2), 1-18.

http://dx.doi.org/10.5354/0719-0581.2016.44556 


\section{Introducción}

La investigación feminista lleva más de medio siglo y varias generaciones académicas abocadas a historizar, desnaturalizar y problematizar la relación entre femineidad y maternidad. No obstante, su estudio permanece muy vigente y se ha vuelto cada vez más complejo y relevante en tanto constituye un fenómeno situado en el campo de lo político, lo social y de la producción de subjetividades.

Durante las últimas décadas, el tradicional binomio mujer-madre, donde la maternidad era prácticamente el sustrato y destino único de la subjetividad femenina, se ha relativizado, acotado y perdido centralidad (Fernández, 1993). De esta manera, algunas mujeres de determinados sectores sociales -medios, profesionales y urbanos- pueden resituar esta experiencia entre otros proyectos de vida y parecieran acceder a mayores grados de elección, en el sentido de poder decidir si ser o no ser madres, cuándo y cómo serlo. Para Donath (2014), los discursos contemporáneos sobre la maternidad se han construido en términos de la dicotomía entre la elección y la no elección. Ser o no ser madre sigue percibiéndose en tanto atributo o carencia, e incluso se manifiesta en términos de posesión y dilema: tener o no tener hijos/as (Zicavo, 2013). La decisión de ser madre se encuentra atravesada por valores sociales ambivalentes entre la obligatoriedad social y el derecho individual (Âlvarez, 2013). El propio discurso de la elección se ha transformado paradójicamente en una obligación cultural profundamente arraigada en normas neoliberales, posfeministas y consumistas, lo que problematiza la construcción de autonomía del género femenino (Imaz, 2014; Lahad, 2014). Para Badinter (2011), además de adherirse o negarse a seguir los mandatos tradicionales de la maternidad, existiría la alternativa de negociar. Esto explicaría la conformación de maternidades bricoleurs (Imaz, 2016), en la que concepciones culturales en apariencia contrapuestas e incompatibles entre sí coexisten caleidoscópicamente entre continuidades, quiebres, innovaciones, contradicciones y paradojas (Imaz, 2016; Lahad, 2014; Zicavo, 2013).

La producción científica latinoamericana también problematiza las tensiones entre antiguos y nuevos mandatos dirigidos hacia las mujeres y sus entrecruzamientos con la maternidad (Burin, 2002; Burin \& Meler, 2010; Fernández, 1993; Molina, 2006; Montilva, 2008; Palomar Verea, 2005; Reid, 2012; Salvo Agoglia \& Gonzálvez Torralba, 2015; Tajer, 2009, 2012; Zicavo, 2013, entre otras). La complejidad de nuestra realidad sociocultural revela la coexistencia de distintos tiempos en lo que respecta al género y la maternidad (Burin \& Meler, 2010). Desde un enfoque que articula los estudios de género con el psicoanálisis, Tajer (2009) acuña la noción de modos de subjetivación de género, clasificándola en formas tradicionales, transicionales e innovadoras para problematizar la heterogeneidad actual en los procesos sociosubjetivos de producción de los géneros. Esto establece nuevas demandas y conflictos específicos, que replantean la interacción entre subjetividad y ordenamiento social, así como la propia noción de deseo y maternidad.

Al igual que en otras latitudes, algunas mujeres latinoamericanas contemporáneas que participan de prácticas públicas y privadas que podrían considerarse innovadoras, especialmente aquellas con carreras profesionales y cuyo ejercicio es una fuente valorada de satisfacciones materiales y simbólicas, conviven con mandatos asociados al buen desempeño en la maternidad (Zicavo, 2013). Si bien estas 
mujeres alcanzan mayores grados de autonomía respecto de las generaciones precedentes, cuando se hace foco en el área de conflicto subjetivo, emergen en primer plano los elevados niveles de estrés, desgaste, culpa, sobreexigencia, como signos de los altos costos que afrontan en el marco de estos procesos de transformación sociocultural (Burin, 2002; Reid, 2012). Para Burin (2002), la noción de deseo de maternidad es uno de los territorios donde actualmente se expresa mayor conflictividad en el campo de la subjetividad femenina. Este deseo se presenta en muchas mujeres como la motivación principal que proporciona una medida homogénea y muchas veces mitificada, y que no otorga lugar a posibles diferencias singulares con respecto a lo que se puede ser o desear (Burin, 2002).

Maternidades emergentes: el caso de las monoparentalidades adoptivas

Un campo fértil para el análisis de las problematizaciones planteadas es el de las monoparentalidades electivas ${ }^{1}$ o las madres solteras por elección ${ }^{2}$ (Mannis, 1999). Este fenómeno ha ganado presencia y visibilización a nivel internacional en las últimas dos décadas en Estados Unidos, Europa y, más recientemente en Latinoamérica. Representa un modelo emergente a través del cual algunas mujeres de alrededor de 40 años de edad, que cuentan mayoritariamente con estudios universitarios y han cumplido con una serie de hitos personales y profesionales, concretan su deseo de ser madres, sin estar en una relación de pareja estable, mediante el uso de técnicas de reproducción asistida (TRA) o de la

\footnotetext{
${ }^{1}$ A lo largo de este artículo se opta por utilizar el término de adopción monoparental para aludir a la situación de adoptar sin pareja, puesto que es la noción de mayor circulación en el medio profesional y académico.

${ }^{2}$ Más recientemente, se está comenzando a estudiar el caso de los padres solteros por elección (PSPE).
}

adopción (Hertz, 2006; Jociles, Rivas, Moncó, Villamil, \& Díaz, 2008; Rivas, Jociles, \& Moncó, 2011; Jociles \& Villaamil, 2012). Dichas características las diferenciarían de las "madres solteras", en el sentido tradicional del término (González, Diez, Jiménez, \& Morgado, 2008; Jociles et al., 2008).

Según González et al. (2008), estas mujeres eligen una maternidad situada en el ámbito del ejercicio libre del propio deseo. No solo desobedecen los mandatos de género convencionales, sino que también se apropian de valores de las sociedades occidentales contemporáneas, como independencia económica, libertad, responsabilidad, elección o realización personal (Jociles \& Villaamil, 2012). Además, realizarían una disociación entre pareja conyugal, pareja parental y pareja progenitora, separando lo hasta ahora unido: sexualidad, procreación, alianza y filiación (Rivas, 2009). Por otra parte, el análisis de las trayectorias vitales y perfiles de estas mujeres las vincula directamente con el fenómeno de la postergación de la maternidad (Montilva, 2008). Este fenómeno puede ser entendido también en un contexto de infertilidad estructural (Marre, 2009) inducida por las condiciones laborales, la dificultad de emancipación de los/as jóvenes, la ausencia de políticas públicas de corresponsabilidad y una cierta forma de violencia simbólica hacia las mujeres que, al retardar o limitar su maternidad, las lleva - no tan electivamente- a recurrir principalmente a las TRA o la adopción para ser madres (Marre, 2009). Justamente ello tensiona el aspecto electivo cristalizado en la propia terminología con la que es nominada este tipo de maternidades.

El estudio de estas monoparentalidades emergentes aún ha sido escasamente abordado por las ciencias sociales latinoameri- 
canas y chilenas (Jociles \& Villaamil, 2012; Salvo Agoglia \& Gonzálvez Torralba, 2015). En particular, las monoparentalidades adoptivas se vuelven una categoría analítica privilegiada por ser una práctica que rompe con diversos principios tradicionales de construcción del parentesco y, al mismo tiempo, recoge tensiones que confirman las formas habituales del ejercicio de la maternidad. Una adopción monoparental es aquella realizada por una persona que no ha formado (no quiso o no pudo) pareja (lo que no le impedirá hacerlo en el futuro) y que, de todas formas, inicia su solicitud a partir de una decisión individual (Giberti, 2010).

Desde la década de 1970 en Estados Unidos y Europa, se incrementó el número de mujeres sin pareja que comenzaron a adoptar, representando entre un $10 \%$ y un $15 \%$ del total de adopciones nacionales e internacionales (González et al., 2008; Haugaard, Palmer, \& Wojslawowicz, 1999; Hertz, 2006; Pakizegi, 2007; Shireman, 1996). En Latinoamérica, estas adopciones han sido posibles más recientemente a partir de algunas modificaciones jurídicas implementadas en algunos países. En Chile, de un total de 510 adopciones realizadas el año 2015, 16 fueron realizadas individualmente, representando un 3,2\% (Servicio Nacional de Menores, Sename, 2015), siendo significativamente inferior a la cifra de adoptantes matrimoniales nacionales e internacionales. Esto puede explicarse tanto por el orden de prelación legal que ubica a las mujeres sin pareja en el último lugar de jerarquía entre los solicitantes, como por otras barreras socioculturales, ideológicas e institucionales $^{3}$ que hacen que muchas interesadas desestimen de antemano emprender este camino o no sean declaradas idóneas.

\footnotetext{
${ }^{3}$ A modo de ejemplo, una de las fundaciones privadas acreditadas no gestiona adopciones no matrimoniales.
}

Algunas autoras del campo antropológico refieren que estas mujeres rompen con concepciones fundadas en paradigmas hondamente biogenéticos del parentesco y relevan aquel que entiende a la maternidad como una experiencia social y vincularmente construida (Jociles Rubio \& Rivas Rivas, 2010; Rivas et al., 2011). Para Rivas et al. (2011), dichas mujeres se vuelven "actores políticos y agentes creadores de nuevos modelos de maternidad, familia y parentesco, en lo que lo central es la maternidad, el deseo de los hijos por sí mismos, sin necesidad de recurrir a la pareja para su legitimización" (p.131). Siguiendo esta línea, según Giberti (2010) se trataría de mujeres que "ejercen autoridad sobre sí mismas pudiendo o no engendrar (debido a su edad) al asumir la maternidad" (p.235). Esto interactúa de forma diversa en el contacto con los otros y con los dispositivos institucionales y profesionales, en donde aún subyace el criterio de que la monoparentalidad per se constituye un factor de riesgo. Esta situación ambigua y oscilante se entrecruza con la percepción que tienen de sí mismas como mujeres empoderadas y autónomas que han podido explorar y perseverar por concretar una maternidad alternativa, con la percepción que los otros tienen de ellas como madres atípicas (Rivas et al., 2011).

De esta forma, puede pensarse que estas maternidades muestran elementos que superan la especificidad del fenómeno y permiten repensar los procesos de construcción de otras maternidades contemporáneas, donde se tensionan significaciones y prácticas tradicionales, transicionales e innovadoras. El objetivo de este artículo es analizar las continuidades y quiebres que se presentan, problematizando nociones como deseo, elección y agencia en este tipo de configuraciones, en el marco de procesos de transformación sociosub- 
jetivos más amplios. Se pretende profundizar en experiencias que tienen la potencialidad de crear nuevos referentes para otras mujeres contemporáneas, con interrogantes como: ¿cuál es el grado de autonomía y de agencia del género femenino para poder actuar de acuerdo a su elección y voluntad en el plano de la maternidad?, ¿podría afirmarse que las adopciones monoparentales constituyen modalidades del todo emancipadas de los mandatos tradicionales respecto de la maternidad?

\section{Metodología}

\section{Enfoque y diseño}

El marco epistemológico y metodológico del estudio fue cualitativo (Vasilachis de Gialdino, 2009) y se optó por un enfoque narrativo (Riessman, 2008), que entiende las historias personales como unidades narrativas constructoras de sentido e identidad, entrelazadas con narrativas sociales y relaciones de poder. La narratividad da cabida a giros biográficos inesperados, permitiendo un proceso permanente de resignificación, lo que habilita conocer las relaciones entre la capacidad de agencia y los discursos y estructuras sociales dominantes, recuperando historias invisibilizadas y periféricas (Riessman, 2008). Se utilizó un diseño exploratorio e interpretativo para contribuir a ampliar un campo de estudio escasamente desarrollado y a comprender en profundidad los significados construidos por las participantes sobre distintos tópicos relacionados con su experiencia adoptiva.

\section{Participantes}

Los criterios de inclusión para participar en el estudio fueron que: 1) la adopción se hubiera realizado entre los años 1999 (fecha de entrada en vigencia de la Ley
$\left.\mathrm{N}^{\circ} 19.620\right)$ y 2012; b) residieran en la Región Metropolitana o en la Quinta Región, por razones de accesibilidad; y c) al momento de ser entrevistadas hubiese transcurrido mínimo un año desde la adopción de su hijo/a. Atendiendo al enfoque de este estudio, la cantidad de participantes no fue determinada por un criterio de saturación, sino por la viabilidad de acceso a las participantes, tomando la decisión de realizar el máximo de entrevistas posibles. El grupo de participantes estuvo conformado por diez mujeres heterosexuales de clase media y media alta que adoptaron sin pareja entre los años 1999 y 2012, en un rango de edad entre 38 y 55 años, con una media de edad de 46 años y residentes de las áreas urbanas mencionadas. Respecto de su estado civil, ocho de ellas eran solteras y dos divorciadas. Al momento de la entrevista, nueve se encontraban sin pareja y una convivía con su pareja heterosexual. Todas manifestaron ser económicamente autónomas, nueve de ellas cursaron estudios universitarios completos y/o de posgrado, y solamente una de ellas contaba con estudios secundarios finalizados. Este perfil mayoritario coincide con el identificado en la producción científica internacional.

Instrumento de producción de información

Se realizó una entrevista narrativa con cada participante (Josselson, Lieblich, \& McAdams, 2003), usando una pauta-guía flexible de preguntas con temas y subtemas a explorar a partir de los objetivos del estudio, sin seguir un orden estricto para dar fluidez a la narración de la entrevistada y al diálogo con la investigadora. Junto con sostener los objetivos de la investigación, fue primordial dejar espacio suficiente para permitir que se desplegara una narrativa significativa, procurando la emergencia de 
historias, ejemplos concretos, episodios o recuerdos (Josselson et al., 2003).

\section{Procedimiento}

El acceso a las participantes se realizó mediante un procedimiento riguroso. Inicialmente, se estableció contacto formal con las jefaturas institucionales de la Fundación Chilena de la Adopción (Fadop) y la Dirección Nacional de Adopciones de Sename, brindándose información detallada de los objetivos del estudio y acordando el carácter de la colaboración, especialmente respecto del apoyo en el contacto con las participantes. Posteriormente, cada institución elaboró listas y realizó un primer contacto con las participantes potenciales con el fin de informarles sobre el estudio, indagar su interés y solicitar su autorización para enviar sus datos de contacto a la investigadora responsable. Luego, la investigadora realizó contactos telefónicos y vía correo electrónico con las interesadas, enviándoles un resumen adaptado del proyecto de investigación y el consentimiento ético informado.

La accesibilidad a las participantes resultó dificultosa por motivos como la falta de actualización de los datos de adopciones más antiguas, lo que imposibilitó la localización de algunas mujeres. En otros casos algunas adoptantes contactadas manifestaron su negativa a participar aduciendo que "ya habían pasado por procesos muy difíciles que necesitaban dejar atrás y alejarse del tema de la adopción", lo que podría evidenciar lo desgastante que resulta el proceso para estas adoptantes; o bien están ejerciendo su derecho legítimo a no querer sentirse objetos de estudio o a resguardar su vida privada. En otros casos, la disponibilidad real estuvo limitada por la falta de tiempo. Por ello, para lograr concretar las entrevistas, la investigadora se acomodó flexiblemente a las posibilidades de las participantes. De esta manera, se entrevistó a seis mujeres mediante el contacto vía institucional, lo que se combinó con la estrategia de bola de nieve, a través de la cual se contactó a las otras cuatro participantes. Se realizó un encuentro con cada entrevistada cuya duración promedio fue de 90 minutos. Todas las entrevistas fueron grabadas y transcriptas.

\section{Análisis de la información}

Se utilizó un método de análisis narrativo (Riessman, 2008), el cual incluye el desarrollo de transcripciones detalladas que examinan el lenguaje, prestando atención al contexto micro y macro de producción, con un enfoque comparativo para identificar similitudes y diferencias entre historias, y atendiendo a cómo las narrativas de cada sujeto reflejan, tensionan o contradicen las narrativas dominantes (Riessman \& Quinney, 2005). Se identificaron núcleos temáticos narrativos y se organizó el material según las relaciones que podían establecerse entre dichos núcleos, que permitieran hallar patrones interpretativos $\mathrm{y}$ establecer significaciones, recurrencias y distinciones en los relatos analizados. Asimismo, se realizó un proceso de triangulación mediante el interanálisis del material producido en una primera instancia de equipo en la Red de Tesistas en Género (Facultad de Psicología, Universidad de Buenos Aires), donde se recogieron nuevos elementos y perspectivas. En una segunda instancia, los resultados fueron presentados a expertos en la temática como forma de validación (Riessman, 2008), lo que favoreció una mayor confiabilidad de los mismos. 


\section{Consideraciones éticas}

Las consideraciones éticas fueron cuidadas transversalmente en el proceso investigativo y mediante el uso de un consentimiento ético informado que fue revisado y aprobado por el Comité de Ética de la Universidad Alberto Hurtado, emitiéndose el respectivo certificado de aprobación. Asimismo, se explicitó claramente a las participantes que, si bien el estudio se realizaba en una fase posadoptiva, no constituía en ningún caso una instancia de seguimiento, en tanto ello era realizado por las instituciones responsables y en el proceso previo de evaluación de idoneidad -particularmente exhaustivo con este perfil de solicitantes- había quedado claramente establecido que disponían de las capacidades requeridas.

\section{Resultados}

Se identificaron tres ejes narrativos significativos ligados a la construcción identitaria, a la construcción de maternidad y a la experiencia de adopción monoparental propiamente tal, los cuales permiten evidenciar la heterogeneidad y pluralidad de las significaciones y prácticas. A continuación, se presentan extractos seleccionados de las entrevistas, utilizando un código para resguardar la confidencialidad.

\section{Narrativas sobre la construcción iden- titaria}

Las participantes de este estudio nacieron entre las décadas de 1960 y 1970, refiriendo en su mayoría haber sido socializadas en familias de sectores urbanos medios y altos que brindaron gran importancia a que estudiaran y lograran un trabajo que promoviera su independencia, sobre todo respecto de los varones. Algunas consideran que fueron fuertemente influenciadas por estas ideas, consideran- do que llevan la delantera en una especie de salto generacional; se ven a sí mismas como mujeres más vanguardistas que sus contemporáneas del entorno familiar, laboral o amistoso. No obstante, refieren que la posibilidad de elegir sus proyectos de vida constituye un derecho que debieron construir con mucho esfuerzo, valentía y perseverancia:

No me someto tan fácil, siempre he sido así, en mi familia habían mandatos que todas mis hermanas cumplieron, y yo nunca sentí que tenía la obligación de hacerlo... Era la que rompía los esquemas porque nunca me ha gustado que las personas tengan la obligación de hacer lo mismo o lo que otros digan, ¿por qué yo no tengo derecho a decidir mi vida? Entonces trataba de tener el derecho. No siempre me fue tan bien, ha sido un camino difícil (E4).

Es la vida que yo elegí... me considero autónoma porque lo he logrado y lo he logrado bien. Creo que hay que ser bastante vanguardista y valiente para poder hacer lo que yo hice (E9).

Lo anterior, se combina también con una sensación de desfase o desencuentro con otras mujeres y varones de su generación, especialmente respecto de quienes tienen alrededor de 50 años actualmente:

Siento que caí en una época desencontrada... a veces tengo la sensación de estar como en una cosa de transición de época (E5).

Asimismo, las entrevistadas reconocen explícitamente las tensiones que las atraviesan, donde coexiste el deseo considerado más convencional de conformar una familia y contar con la manutención económica masculina junto con el deseo de desarrollar mayor autonomía, indepen- 
dencia económica y crecer profesionalmente:

Yo te diría que tengo un ala súper conservadora en mi forma de vida, pero al mismo tiempo, me siento muy vanguardista respecto de otras mujeres de mi época, soy muy súper abierta a la diversidad (E2).

Narrativas sobre la construcción de maternidad

Las narrativas de las participantes muestran que, si bien muchas de ellas significan la maternidad como fruto de una elección libre y personal, aparece simultáneamente en sus relatos una especie de fuerza o instinto que hace que necesiten ser madres como parte de la naturaleza femenina:

Siempre desde que era niña cuando pensaba en esos sueños de grande, siempre quise tener un hijo (E1).

La maternidad no es una cuestión conceptual sino que es vivencial, es como el llamado de la selva no más (E8).

Asimismo, emergen narrativas que muestran que la maternidad es un eje central de la identidad. Una entrevistada que presentó un diagnóstico de menopausia precoz muestra en su relato la centralidad de la maternidad en su construcción identitaria y el impacto subjetivo que tuvo para ella la imposibilidad biológica de su realización, al punto de concebir que una parte de sí misma se moría:

En esa época yo recuerdo que lo pasé mal, muy mal. Siento que una parte de mi identidad de mujer se murió con eso, de verdad. Dejé de ser mujer, sentí que no podía entregar al mundo lo que se suponía que una mujer tenía que entregar (E5).

Por otra parte, sus trayectorias vitales evidencian claramente el fenómeno de postergación de la maternidad, luego de concretar otros proyectos que tuvieron prioridad. La mayoría de las entrevistadas expresan en sus relatos la dificultad que tuvieron de conciliar su deseo de maternidad con su carrera profesional y, al mismo tiempo, el deseo de construir una vida autónoma e independiente que les permitiera elegir con madurez, intención y reflexivamente, el momento y la forma de ser madres, lo que en la práctica implicó la vivencia de una maternidad tardía:

Comencé a trabajar sumamente joven y logré todas mis metas profesionales... Tengo varios posgrados y me realicé plenamente en lo profesional, pero me faltaba la parte mamá (E10).

En los relatos se muestra un punto de inflexión, fuertemente relacionado con el reloj biológico y social, y con la energía requerida para criar y cuidar un hijo/a, en el que definen que ya es el momento de concretar el proyecto de maternidad y que dicho proyecto no será más postergado y tendrá prioridad frente a otros, como por ejemplo, la búsqueda de una pareja:

Llegado el momento me dije: "Para encontrar la pareja tengo muchos años por delante y puede ser en cualquier momento de la vida, pero para la maternidad no". ¿Y me lo quiero perder? No, no me lo quiero perder (E9).

La elección de otros caminos no convencionales para construir una familia emerge como una forma de concretar su deseo de maternidad, al cual no están dispuestas a renunciar bajo ninguna circunstancia, 
aun cuando no se haya dado la oportunidad de estar en pareja:

Mi sueño de origen era absolutamente de Susanita ${ }^{4} \ldots$ lo que pasa es que no se dieron las cosas y como no se dieron las cosas decidí que yo no me iba a echar a morir por eso y que no iba a renunciar a algo que para mí era tan importante en la vida como la maternidad, porque no se daba la condición clásica de estar en pareja, ese fue el punto de quiebre (E8).

En los relatos de las entrevistadas se observa que la maternidad es vivida como un proceso reflexivo, en el que la intencionalidad se pone en juego al momento de informarse, tomar la decisión, y luego comunicarla y argumentarla ante los demás. Así, esta decisión emerge como resultado de un proceso de autoconocimiento en el que se plantea esta alternativa y los diversos desafíos implicados, hasta alcanzar un cierto grado de certeza para dar los pasos siguientes:

Me di un tiempo para pensarlo seriamente conmigo misma, sin a contarle nadie esto... ¿vas a ir a comunicárselo al mundo? Porque tienes que estar segura cuando lo comuniques (E5).

En cuanto al momento de comunicar a su entorno la decisión de adoptar, al igual que en otros estudios (González et al., 2008; Rivas et al., 2011), la mayoría refiere que lo hicieron con la decisión tomada. Las razones esgrimidas para no comunicarlo desde un primer momento son el manejo de la posible frustración que podía conllevar el proceso y la dificultad adicional que implicaría generar

\footnotetext{
${ }^{4}$ Personaje de la caricatura Mafalda, que representa a la mujer cuyo mayor deseo es casarse con el príncipe azul, ser dueña de casa y tener muchos hijos.
}

expectativas tanto para sí mismas como para quienes las rodean:

No quería crearles expectativas a todo el mundo porque en el fondo también me las iba a crear yo. $\mathrm{Y}$ yo quería ser bien realista porque me podían decir que no perfectamente $(\mathrm{E} 4)$.

A diferencia de la mayoría, otra entrevistada relató que antes de tomar la decisión e iniciar la solicitud de adopción, decidió conversarlo con su familia para indagar los apoyos con los que contaría en una futura adopción. Al respecto argumentó que para ella era un requisito clave que su entorno estuviera a favor de la adopción para prevenir situaciones de discriminación o de trato diferencial hacia un futuro hijo/a:

Yo antes de dirigirme a la fundación a iniciar todo este proceso, lo conversé con todos. Porque ¿qué sacaba yo con ser mamá adoptiva si al final uno va a vivir en una familia?, ¿si vamos a estar sufriendo?... porque yo puedo querer ser mamá y convertirme en mamá, pero si a mi hija no la van a aceptar, ¿qué sacamos? (E1).

Otra de las razones aludidas por las entrevistadas para adoptar dice relación con una lógica de autorrealización, en la que se destacan aquellas emociones y sentimientos positivos vividos desde que son madres, emergiendo relatos marcados por la felicidad y la plenitud:

La gratificación personal es mucha, es maravilloso, una experiencia increíble (E8).

Junto a lo anterior, se identifican narrativas que conciben la adopción como un logro tras enfrentar con mucho esfuerzo, perseverancia y paciencia este camino, lo que se cruza con una autodefinición de personas 
capaces de enfrentar las pruebas que implica el proceso adoptivo para ellas:

Durante todo el tiempo que yo luché por la adopción, mis familiares y amistades, me insistían que desistiera frente a cada problema que surgía. Cada cortafuego que aparecía y me ponían en el sistema. Me decían: "Ya déjalo, si tú tienes que resignarte a la voluntad de Dios que no tengas hijos. No te casaste y ya no, no más", pero yo nunca me resigné (E4).

De esta manera, adoptar constituye para muchas el logro de una serie de metas asociadas a cada etapa del proceso adoptivo, desde la decisión en adelante. Un caso ilustrativo del esfuerzo realizado es el de una entrevistada que decidió y logró terminar sus estudios secundarios para cumplir con los requisitos legales exigidos para adoptar:

No tenía el cuarto medio entonces tuve que estudiar paralelamente, hice los estudios y exámenes libres, me preparé y di las pruebas que aprobé afortunadamente (E6).

Llegado el momento de ser madres, se muestra la incidencia que ello tiene en sus carreras profesionales y laborales y las estrategias desarrolladas para enfrentar el desafío de la conciliación. Algunas participantes refieren la presencia de un discurso social que les advierte los altos costos asociados a la maternidad, así como sus respuestas a esto, concibiendo como implícito a toda maternidad responder a múltiples demandas y aprender a realizar algunas renuncias:

Me planteaban las dificultades que implica un niño, que tienes que estar pendiente, enfermedades, dificultades escolares, ropa, alimentación, salud. Y mi planteo era si ser mamá implica eso, o sea, no existe otra forma de ser mamá. Nadie puede formar familia de otra manera sin hacerse cargo de sus hijos, si no cuéntame quién y que me pase la receta. Entonces yo me planteé la renuncia a un modo de vida de profesional libre e independiente (E5).

Otro caso muestra claramente la opción por un modelo de maternidad intensiva y exclusiva (Hays, 1998), en el que la entrevistada decidió jubilarse anticipadamente para dedicarse a tiempo completo a las tareas de la maternidad, decisión que significa como una elección que le genera bienestar y no como renuncia o sacrificio:

Dejé de trabajar para ser mamá. Me jubilé anticipadamente para tener la oportunidad de ser mamá... yo quería hace muchos años adoptar y mi trabajo no lo permitía porque cumplía muchos turnos y yo quería ser mamá de tiempo completo. Nunca me he arrepentido, estoy feliz (E10).

Otras entrevistadas consideran que la renuncia no necesariamente es una condición sine qua non a la maternidad y que es necesario aprender a equilibrarla con otros ámbitos de la vida, para que la identidad no quede completamente capturada por la maternidad:

Es necesario equilibrar el espacio personal e individual de mujer también. No transformarse exclusivamente en mamá, que tu identidad no sea solamente la maternidad (E8).

Narrativas sobre la adopción monoparental

La totalidad de las entrevistadas enfatizan que la adopción siempre fue una alternativa muy valorada tempranamente en sus 
vidas, más allá de estar o no en pareja o presentar problemas de fertilidad, asociándola siempre con sentimientos gratificantes y con un sentimiento de coherencia valórica e ideológica:

Es loco lo que te voy a decir, pero en mi corazón yo siempre supe que iba a ser madre adoptiva, desde pequeña decía: "si tengo hijos, voy a adoptar", o sea, eso estuvo desde siempre en mi cabeza (E7).

En sus relatos sitúan la adopción como la primera opción entre otras alternativas. En algunos casos, y aun disponiendo de medios económicos, refieren no haber recurrido a TRA por decisión propia. Las razones esgrimidas para descartar esta vía son coincidentes con las de otros estudios que identifican como argumentos de descarte el cuestionamiento a la lógica de mercado, la artificialidad y la no explicabilidad, debido a la dificultad de poder construir un relato apropiado respecto del origen de sus hijos/as, en coherencia con diferentes sistemas valóricos que jerarquizan las diferentes vías para ser madres en el caso de mujeres solteras (Jociles Rubio \& Rivas Rivas, 2010):

Si tú no estás con pareja tienes que comprar el semen. Entonces entras en una dinámica de niño de supermercado... eso para mí es lo más antiético y antinatural que existe para tener un hijo (E2).

Me pasó sentir que la inseminación no era para mí... ¿qué historia le construyes a ese niño? Yo creo que la tendría que haber aprendido de memoria, no me saldría decir: "Estaba sola, quería tener un hijo y te traje a ti". Me parecía muy de laboratorio, no, no, no me gustó (E5).
Respecto de la fecundación sexual con un desconocido, otra entrevistada esgrime argumentos de tipo ético vinculados a evitar el engaño y la instrumentalización de otra persona (Jociles \& Rivas, 2010; Jociles \& Villaamil, 2012) y a poder contar una historia ligada con el amor:

Los hijos tienen que venir de un proceso de amor. Uno no puede meterse con un tipo y no decirle nada y quedarse embarazada... habría sido facilísimo buscar un semental, pero cómo miras a tu hijo a los ojos después y le dices: me fui a meter con cualquier persona para poder tenerte (E6).

Por otra parte, algunas adoptantes tienden a igualar su maternidad adoptiva con la maternidad convencionalmente entendida, cuestionando los paradigmas biologicistas del parentesco y enfatizando el carácter de construcción social de la maternidad y la filiación:

Igual que cualquier guagua [bebé] que nace. A mí me dijeron: "Esta va a ser tu hija" y punto (E10).

Junto con ello, aparecen ideas que desmitifican que la experiencia física del embarazo sea un elemento que diferencie estas maternidades. La única adoptante con un hijo de origen biológico antes de adoptar refiere:

No haberla tenido en la guata [panza o barriga] es la única cosa que te podría decir que es diferente... porque el parto es muy semejante, digamos a lo que yo sentí cuando tuve a mi hijo (biológico) a cuando me entregaron a mi hija (adoptiva) (E3). 


\section{Discusión y conclusiones}

Sin el afán de generalizar los resultados, el análisis de los relatos evidencia dinamismos que permiten problematizar que no existen relaciones dicotómicas o absolutas en lo referido a la elección, autonomía o agencia, tanto en esta práctica de maternidad, como en ninguna otra. Retomando la noción de modos de subjetivación de género (Tajer, 2009), sus experiencias y proyectos de maternidad se ven atravesadas por las tensiones propias de un contexto de transformaciones; y están situadas en una interfaz donde conviven modos de subjetivación femeninos tradicionales, transicionales e innovadores. Al igual que todo sujeto, estas mujeres cuentan con grados de autonomía relativos en función de los límites impuestos por contextos históricos, sociales, económicos y políticos determinados (Tubert, 2001). En consecuencia, solo es posible pensar la elección como un significante multivalente dependiente de los dinámicos contextos sociales, relacionales y subjetivos (Lahad, 2014). Y la capacidad de agencia involucra cuotas variables de autonomía, reflexividad y creatividad que permiten ampliar los márgenes de acción más allá de la mera reproducción estructural (Yopo Díaz, 2016).

Tomando los diversos posicionamientos de autoras anteriormente referenciadas (Álvarez, 2013; González et al., 2008; Jociles et al., 2008; Jociles \& Villaamil, 2012; Rivas, 2009; Rivas et al., 2011; Zicavo, 2013), podría considerarse que estas elecciones no son absolutamente libres y que la decisión de llevar adelante este proyecto se encuentre atravesada tanto por el deseo como por el mandato social, en tanto el deseo es una construcción sociosubjetiva imposible de desligar del contexto cultural y epocal, cuestión que es transversal a toda experiencia de parentalidad.

Estas mujeres cuentan con mayores grados de autonomía, elección y agencia, dado que han podido desear y concretar otros múltiples proyectos y no ser únicamente madres. Sus nuevas prácticas no necesariamente son acompañadas de una problematización de los supuestos tradicionales en torno a la maternidad, la familia y/o el desarrollo profesional; tampoco la posibilidad de pensar la no-maternidad, cuestión que las generaciones más jóvenes sí se están planteando. Todas expresan que la maternidad ha sido un referente principal de autorrealización personal y un proyecto de vida irrenunciable que han deseado alcanzar en momentos más tardíos de la vida y estando dispuestas a traspasar muchas pruebas para lograrlo. Asimismo, en algunas entrevistadas subsiste la idea de maternidad intensiva y exclusiva (Hays, 1998).

En cuanto a la noción de deseo de maternidad, considerando que este no fue un estudio clínico, el análisis individual de cómo se configura en cada participante escapa a los propósitos y alcances del mismo. No obstante, es posible observar en sus narrativas, al igual que en otras maternidades contemporáneas, modalidades deseantes atravesadas por una amalgama de anhelos narcisistas y de continuidad, mandatos sociales y familiares, representaciones, elecciones, oportunidades, entre otros elementos. En cada maternidad se entramará singularmente el deseo inconsciente/consciente de cada mujer, las relaciones de parentesco en unas condiciones histórico-sociales determinadas, la organización de la cultura patriarcal (Tubert, 2001), así como su propia historia individual. Este deseo no debe ser entendido entonces en términos 
dicotómicos de presencia/ausencia ni como sustituto compensatorio de una carencia primordial, sino que puede ser reconceptualizado como producción o potencia (Fernández, 2013).

Coincidentemente con los hallazgos de González et al. (2008), las entrevistadas se manifiestan altamente satisfechas y realizadas con la adopción, ya que gracias a esta experiencia han alcanzado uno de sus deseos prioritarios de autorrealización. Solamente mencionan de forma tangencial el desgaste y las múltiples exigencias que enfrentan cotidianamente, viéndolas como el resultado lógico de sus elecciones. Esto puede estar parcialmente influenciado por un factor de deseabilidad social inherente a toda investigación cualitativa, acentuado por el hecho de ser mujeres expuestas a una mirada social e institucional evaluativa frente a la que legitiman su opción. Se hipotetiza que visibilizar algún grado de malestar o de vulnerabilidad puede ser leído por las propias entrevistadas como una exposición al cuestionamiento de su proyecto o de sus capacidades. Además, pareciera constituir un punto ciego la visibilización de elementos de orden sociocultural que han impactado en sus trayectorias y decisiones vitales que las hicieron postergar su maternidad, o la ausencia de políticas públicas que les impiden alcanzar mayores niveles de conciliación entre diferentes ámbitos de sus vidas, quedando la solución siempre y casi exclusivamente en sus manos.

En consonancia con hallazgos de otros estudios, parte de los elementos anteriores pueden ser comprendidos como estrategias de "camuflaje" (Rivas et al., 2011), en las que se opta por no problematizar mayormente diversos aspectos y se acude a discursos políticamente correctos al hablar de la maternidad o exponer sus razones para adoptar. El objetivo es evitar que su opción sea vista como fruto de alguna carencia (ser mayores, presentar problemas reproductivos o no haber conseguido pareja con la cual tener hijos biológicos, etc.). Se debe considerar además que el deseo de maternidad es la motivación considerada más funcional para el discurso experto en los procesos evaluativos de idoneidad (Jociles \& Charro, 2008), lo que podría explicar por qué muchas de ellas utilizan recurrentemente en sus relatos elementos ideológicos convencionales que sitúan a la maternidad como eje de la identidad femenina (Jociles, Rivas, \& Poveda, 2012), y como experiencia que les brinda una total autorrealización (González et al., 2008).

No obstante, otros relatos evidencian su análisis crítico y reflexivo de los mandatos de género, la maternidad y la familia, mostrándose muy lúcidas en la identificación y la interpretación de las contradicciones culturales que deben gestionar en sus experiencias cotidianas con diversas personas y espacios sociales. Muestran así su capacidad permanente de agencia en la construcción y reconstrucción de nuevos sentidos y prácticas de parentesco, desligadas de lo biológico y de la vida en pareja. Y, en la gestión micropolítica de las tensiones y desafíos del día a día, construyen respuestas originales que van abriendo camino a la resignificación. Sin embargo, a diferencia de otros países, muchas de estas innovaciones quedan adheridas al terreno de lo privado; en el contexto chileno aún no se advierte la conformación de agrupaciones o espacios más informales de asociatividad e intercambio entre adoptantes monoparentales, como por ejemplo blogs (Hertz, Rivas, \& Rubio Jociles, 2016). En este sentido, un pequeño paso para su agencia puede ser que accedieran a participar de este estudio, argumentando su interés en relatar su 
vivencia a modo de testimonio para que las instituciones, profesionales y otros actores sociales puedan interrogar sus premisas y prácticas, y para llegar a otras mujeres que se estén planteando tomar este camino, dándose de facto una transferencia de experiencias que, progresivamente, vaya fracturando los modelos familiares hegemónicos.

Estos resultados y problematizaciones pretenden contextualizar el escenario dinámico de transformaciones en el cual las elecciones y proyectos de vida de las mujeres aludidas discurren. La licitud plasmada en el orden de prelación de la normativa chilena vigente, la validación diferencial que se hace desde las ideologías dominantes y la relación establecida con algunas instituciones y profesionales del campo psicosocial continúan poniendo bajo sospecha o en un lugar de menor estatus estas maternidades alternativas. Enfrentados a una época de profundas transformaciones, es un imperativo ético interpelar diversas nociones ampliamente aceptadas en los dispositivos institucionales que regulan quiénes pueden o no ejercer la parentalidad. Los modelos conceptuales de referencia, las convicciones y los prejuicios que influencian el setting de encuentro con los/las adoptantes deben ser objeto de elaboración para no impedir a los operadores acoger a las personas reales que tienen en frente (D'Andrea, 2009); y para que, como muy lúcidamente expresa Théry (2009), "estas familias no corran el riesgo de pagar las consecuencias de nuestras incertidumbres, al tratarlas como chivos expiatorios de las inquietudes de nuestra época" (p. 41).

En vista de lo anterior, es clave continuar realizando estudios que contextualicen y profundicen el conocimiento y la comprensión de los deseos, elecciones, sentidos y prácticas que emergen por fuera de los modelos hegemónicos patriarcales para que los prejuicios o las conceptualizaciones no nos hagan significar representaciones y prácticas inéditas como psicopatológicas (Reid, 2012). Al mismo tiempo, se requiere construir criterios para identificar las formas que puede adoptar lo psicopatológico en lo nuevo (Tajer, 2012). La escucha profesional debe contemplar nuevas y actualizadas coordenadas políticas, sociales y conceptuales, atendiendo a la singularidad emergente para colaborar en la construcción de espacios evaluativos y de acompañamiento que alojen las propias reflexiones de estas solicitantes, respecto de los caminos y motivaciones que las llevan a esta decisión, así como el análisis sobre las posibilidades y desafíos vinculados. Las maternidades contemporáneas y emergentes requieren ser situadas en un espacio de preguntas abiertas en el marco de un continuo proceso de deconstrucción, resignificación y creación.

Para finalizar, es posible identificar como limitaciones del estudio su foco solamente en uno de los actores involucrados en este proceso. Por tanto, como proyecciones de esta línea de investigación, se vuelve muy necesario conocer las voces de otros actores que tienen incidencia sobre estos procesos, como operadores psicosociales, autoridades administrativas, judiciales y legisladores, y ciudadanos en general. Además, resulta fundamental tomar la perspectiva de los propios niños, niñas y adolescentes que forman parte de estas familias, cuestión que ha sido escasamente explorada en el estudio de la diversidad familiar. Asimismo, resultaría interesante explorar las experiencias novedosas de los primeros varones que han realizado adopciones sin pareja en Chile o que están comenzando a acercarse a las instituciones a buscar información. 


\section{Referencias}

Álvarez, B. (2013). La maternidad: entre la decisión individual y/o la obligatoriedad social. En C. López, D. Marre, \& J. Bestard (Eds.), Maternidades, procreación y crianza en transformación (pp. 219-244). Barcelona, España: Edicions Bellaterra.

Badinter, E. (2011). La mujer y la madre. Un libro polémico sobre la maternidad como nueva forma de esclavitud. Madrid, España: La Esfera de los Libros.

Burin, M. (2002). Estudios sobre la subjetividad femenina. Mujeres y salud mental. Buenos Aires, Argentina: Librería de Mujeres Editoras.

Burin, M. \& Meler, I. (2010). Género y familia. Poder, amor y sexualidad en la construcción de la subjetividad. Buenos Aires, Argentina: Paidós.

D’Andrea, A. (2009). Los desafíos evolutivos de la familia adoptiva. Psicoperspectivas, $8(1), 159-194$. Recuperado de http://www.psicoperspectivas.cl/index.php/psicoperspectivas/article/viewFile/75/61

Donath, O. (2014). Choosing motherhood? Agency and regret within reproduction and mothering retrospective accounts. Women's Studies International Forum, 53, 200-209. http://dx.doi.org/10.1016/j.wsif.2014.10.023

Fernández, A. M. (1993). La mujer de la ilusión: pactos y contratos entre hombres y mujeres. Buenos Aires, Argentina: Paidós.

Fernández, A. M. (2013). Jóvenes de vidas grises. Psicoanálisis y biopolítica. Buenos Aires, Argentina: Nueva Visión.

Giberti, E. (2010). Adopción siglo XXI. Leyes y deseos. Buenos Aires, Argentina: Editorial Sudamericana.

González, M.-M., Diez, M., Jiménez, I., \& Morgado, B. (2008). Maternidad a solas por elección: primera aproximación. Anuario de Psicología, 39(1), 119-126. Recuperado de http://www.raco.cat/index.php/anuariopsicologia/article/viewFile/99375/159767

Haugaard, J. J., Palmer, M., \& Wojslawowicz, J. C. (1999). Single-parent adoptions. Adoption Quarterly, 2(4), 65-74.

http://dx.doi.org/10.1300/J145v02n04_05

Hays, S. (1998). Las contradicciones culturales de la maternidad. Barcelona, España: Paidós.

Hertz, R. (2006). Single by chance, mothers by choice. New York, New York: Oxford University Press.

Hertz, R., Rivas, A. M., \& Rubio Jociles, M. I. (2016). Single mothers by choice in Spain and the United States. En C. L. Shehan (Ed.), The Wiley Blackwell encyclopedia of family studies (pp. 1-5). New York, New York: John Wiley \& Sons.

http://dx.doi.org/10.1002/9781119085621.wbefs005

Imaz, M. E. (2014). Maternidades lesbianas, nuevas formas familiares y derecho a elegir.

Grafo. Working Papers, 3(2), 1-12. Recuperado de

http://revistes.uab.cat/grafowp/article/view/v3-imaz 
Imaz, E. (2016). Las madres bricoleurs. Estrategias, prácticas y modelos maternales contemporáneos. Estudos Feministas, 24(2), 485-497.

http://dx.doi.org/10.1590/1805-9584-2016v24n2p485

Jociles, M. I. \& Charro, C. (2008). Construcción de los roles paternos en los procesos de adopción internacional: el papel de las instituciones intermediarias. Política y Sociedad, 45(2), 105-130. Recuperado de http://revistas.ucm.es/index.php/POSO/article/view/POSO0808230105A

Jociles, M. I., Rivas, A. M., Moncó, B., \& Villaamil, F. (2010). Madres solteras por elección: entre el "engaño" y la solidaridad. Revista de Antropología Iberoamericana, 5(2), 256-299. Recuperado de http://www.redalyc.org/articulo.oa?id=62319343005

Jociles, M. I., Rivas, A. M, Moncó, B., Villamil, F., \& Díaz, P. (2008). Una reflexión crítica sobre la monoparentalidad: el caso de las madres solteras por elección. Revista Portularia, 8(1), 265-274. Recuperado de http://rabida.uhu.es/dspace/handle/10272/2186

Jociles, M. I., Rivas, A. M., \& Poveda, D. (2012). Las representaciones expertas sobre las solicitantes individuales en los procesos de adopción. Revista de Dialectología y Tradiciones Populares, 67(2), 535-558.

http://dx.doi.org/10.3989/rdtp.2012.21

Jociles, M. I. \& Villaamil, F. (2012). Madres solteras por elección: representaciones sobre la fecundación sexual como vía de acceso a la maternidad. Chungará, Revista de Antropología Chilena 44(4), 717-731. Recuperado de

http://www.scielo.cl/pdf/chungara/v44n4/art12.pdf

Jociles Rubio, M. I. \& Rivas Rivas, A. M. (2010). Motivaciones genéticas y experienciales: el discurso de las MSPE sobre la fecundación asistida como vía de acceso a la maternidad en solitario. Alteridades, 20(39), 107-124. Recuperado de https://is.gd/T9D8gn

Josselson, R, Lieblich, A., \& McAdams, D. P. (Eds.). (2003). Up close and personal: The teaching and learning of narrative research. Washington, District of Columbia: American Psychological Association.

Lahad, K. (2014). The single woman's choice as a zero-sum game. Cultural Studies, 28(2), 240-266.

http://dx.doi.org/10.1080/09502386.2013.798341

Mannis, V. S. (1999). Single mothers by choice. Journal of Family Relations, 48(2), 121-128. http://dx.doi.org/10.2307/585075

Marre, D. (2009). Los silencios de la adopción. Revista de Antropología Social, 18, 97-126. Recuperado de http://revistas.ucm.es/index.php/RASO/article/view/RASO0909110097A/8841

Molina, M. E. (2006).Transformaciones histórico culturales del concepto de maternidad y sus repercusiones en la identidad de la mujer. Psykhe, 15(2), 93-103. http://dx.doi.org/10.4067/S0718-22282006000200009 
Montilva, M. (2008). Postergación de la maternidad de mujeres profesionales jóvenes en dos metrópolis latinoamericanas. Utopía y Praxis Latinoamericana, 13(41), 69-79. Recuperado de

http://www.redalyc.org/articulo.oa?id=27904104

Pakizegi, B. (2007). Single-parent adoptions and clinical implications. En J. Rafael Art, A. Baden, F. Biafora, \& A. Camacho-Gingerich (Eds.), Handbook of adoption. Implications for researchers, practitioners, and families (pp. 190-216). Thousand Oaks, California: Sage.

Palomar Verea, C. (2005). Maternidad: historia y cultura. Revista de Estudios de Género. La Ventana, 22, 35-67. Recuperado de http://www.redalyc.org/articulo.oa?id=88402204

Reid, G. (2012). Ninguna y todas quieren ser madres hoy. Nuevas prácticas, nuevas representaciones de la maternidad. En D. Tajer (Comp.), Género y salud. Las políticas en acción (pp.119-136). Buenos Aires, Argentina: Lugar Editorial.

Riessman, C. K. (2008). Narrative methods for the human sciences. London, United Kingdom: Sage.

Riessman, C. K. \& Quinney, L. (2005). Narrative social work. A critical review. Qualitative Social Work, 4(4), 391-412.

https://dx.doi.org/10.1177/1473325005058643

Rivas, A. (2009). Pluriparentalidades y parentescos electivos. Revista de Antropología Social, 18, 7-19. Recuperado de

http://revistas.ucm.es/index.php/RASO/article/viewFile/RASO0909110007A/8801

Rivas, A. M., Jociles, M. I., \& Moncó, B. (2011). Las madres solteras por elección. ¿Ciudadanas de primera y madres de segunda? Revista Internacional de Sociología, 69(1), 121-142. Recuperado de

http://revintsociologia.revistas.csic.es/index.php/revintsociologia/article/viewArticle/360

Salvo Agoglia, I. \& Gonzálvez Torralba, H. (2015). Monoparentalidades electivas en Chile: emergencias, tensiones y perspectivas. Psicoperspectivas, 14(2), 40-50. Recuperado de http://www.scielo.cl/scielo.php?script=sci_arttext\&pid=S0718-69242015000200005

Servicio Nacional de Menores, Sename. (2015). Anuario estadístico institucional. Santiago, Chile: Servicio Nacional de Menores, Gobierno de Chile. Recuperado de http://www.sename.cl/wsename/images/anuario_2015_final_200616.pdf

Shireman, J. F. (1996). Single parent adoptive homes. Children and Youth Services Review, $18(1-2), 23-36$.

http://dx.doi.org/10.1016/0190-7409(95)00052-6

Tajer, D. (2009). Heridos corazones. Vulnerabilidad coronaria en varones y mujeres. Buenos Aires, Argentina: Paidós.

Tajer, D. (2012). Notas para una práctica psicoanalítica postpatriarcal y postheterónima. En M. Burin, I. Meler, D. Tajer, J. C. Volnovich, \& C. Hazaki (Comp.), La crisis del patriarcado (pp.79-92). Buenos Aires, Argentina: Topía Editorial. 
Théry, I. (2009). El anonimato en las donaciones de engendramiento: filiación e identidad narrativa infantil en tiempos de descasamiento. Revista de Antropología Social, 18, 21 42. Recuperado de http://revistas.ucm.es/index.php/RASO/article/download/RASO0909110021A/8807

Tubert, S. (2001). Deseo y representación. Convergencias de psicoanálisis y teoría feminista. Madrid, España: Editorial Síntesis.

Vasilachis de Gialdino, I. (2009). Estrategias de investigación cualitativa. Buenos Aires, Argentina: Gedisa.

Yopo Díaz, M. (2016). El tiempo de las mujeres en Chile: repensar la agencia. Revista de Estudios Sociales, 57, 100-109.

http://dx.doi.org/10.7440/res57.2016.08

Zicavo, E. (2013). Dilemas de la maternidad en la actualidad: antiguos y nuevos mandatos en mujeres profesionales de la ciudad de Buenos Aires. Revista de Estudios de Género La ventana, 4(38), 50-87. Recuperado de http://www.redalyc.org/pdf/884/88430445004.pdf 